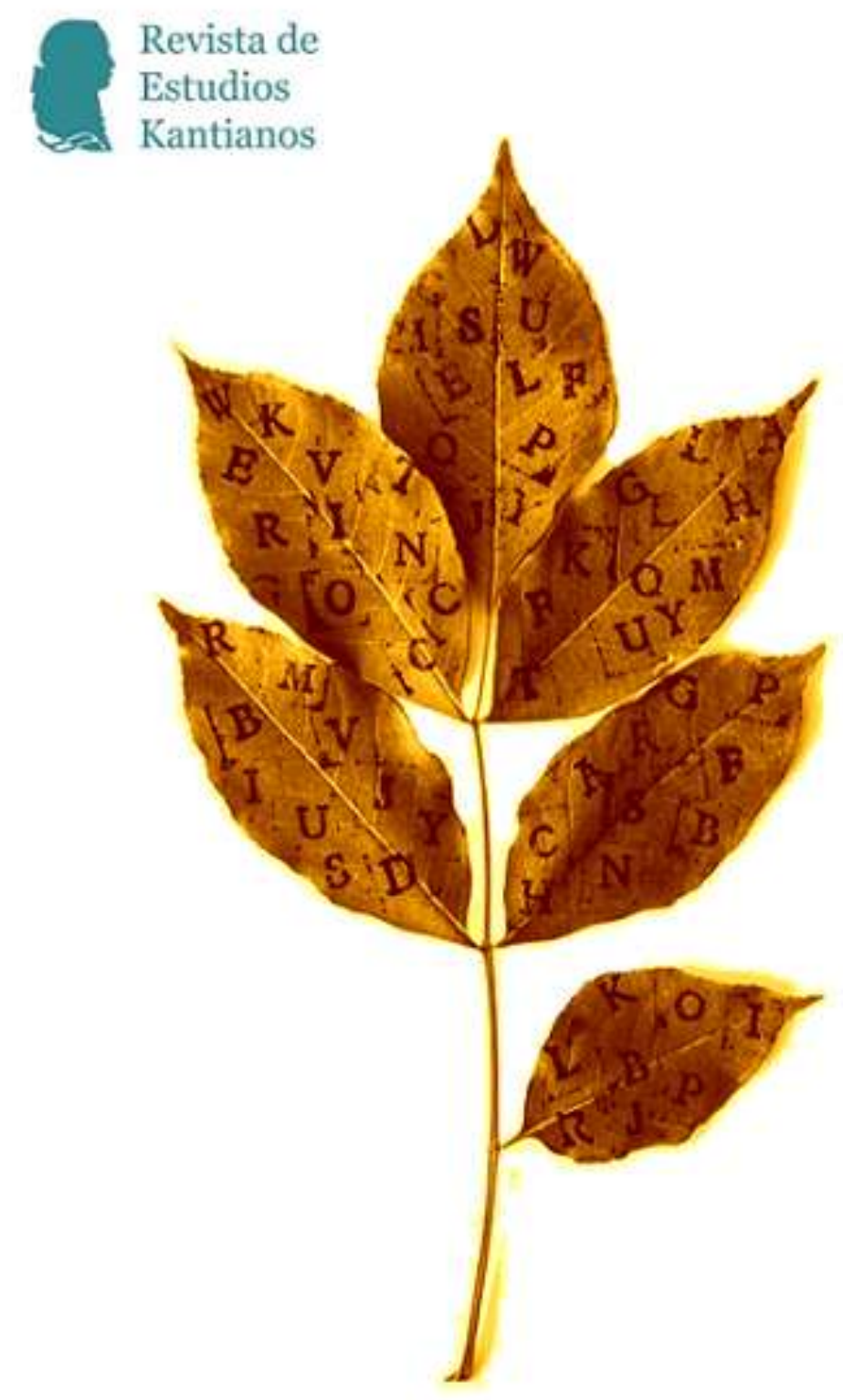




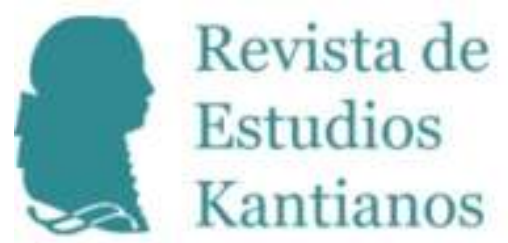




\section{Revista de Estudios Kantianos}

Publicación internacional de la Sociedad de Estudios Kantianos en Lengua Española Internationale Zeitschrift der Gesellschaft für Kant-Studien in Spanischer Sprache International Journal of the Society of Kantian Studies in the Spanish Language

\section{Dirección}

Pedro Jesús Teruel, Universitat de València

pedro.teruel@uv.es

Hernán Pringe, CONICET-Universidad de Buenos Aires/

Universidad Diego Portales, Santiago de Chile

hpringe@gmail.com

\section{Secretario de edición}

Fernando Moledo, Universidad de Buenos Aires - CONICET

fernandomoledo@gmail.com

\section{Secretaria de calidad}

Marcela García, Instituto de Investigaciones Filosóficas, UNAM

garciar.marcela@gmail.com

\section{Editores científicos}

Jacinto Rivera de Rosales, UNED, Madrid

Claudia Jáuregui, Universidad de Buenos Aires

Vicente Durán, Pontificia Universidad Javeriana, Bogotá

Julio del Valle, Pontificia Universidad Católica del Perú, Lima

Jesús Conill, Universitat de València

Gustavo Leyva, Universidad Autónoma de México, México D. F.

María Xesús Vázquez Lobeiras, Universidade de Santiago de Compostela

Wilson Herrera, Universidad del Rosario, Bogotá

Pablo Oyarzun, Universidad de Chile, Santiago de Chile

Paula Órdenes Azúa, Universität Heidelberg 


\section{Comité científico}

Juan Arana, Universidad de Sevilla

Reinhardt Brandt, Philipps-Universität Marburg

Mario Caimi, Universidad de Buenos Aires

Monique Castillo, Université de Paris-Est

Adela Cortina, Universitat de València

Bernd Dörflinger, Universität Trier

Norbert Fischer, Universität Eichstätt-Ingolstadt

Miguel Giusti, Pontificia Universidad Católica del Perú

Dulce María Granja, Universidad Nacional Autónoma de México

Christian Hamm, Universidad Federal de Santa María, Brasil

Dietmar Heidemann, Université du Luxembourg

Otfried Höffe, Universität Tübingen

Claudio La Rocca, Università degli Studi di Genova

Juan Manuel Navarro Cordón, Universidad Complutense, Madrid

Carlos Pereda, Universidad Nacional Autónoma de México

Gustavo Pereira, Universidad de la República, Uruguay

Ubirajara Rancan de Azevedo, Universidade Estadual Paulista, Brasil

Margit Ruffing, Johannes Gutenberg-Universität Mainz

Gustavo Sarmiento, Universidad Simón Bolívar, Venezuela

Sergio Sevilla, Universitat de València

Roberto Torretti, Universidad Diego Portales, Santiago de Chile

Violetta Waibel, Universität Wien

Howard Williams, University of Aberystwyth

Allen W. Wood, Indiana University

Diseño, revisión de estilo y maqueta

Josefa Ros Velasco, Universidad Complutense de Madrid

\section{Entidades colaboradoras}

Sociedad de Estudios Kantianos en Lengua Española (SEKLE)

Departament de Filosofia de la Universitat de València 


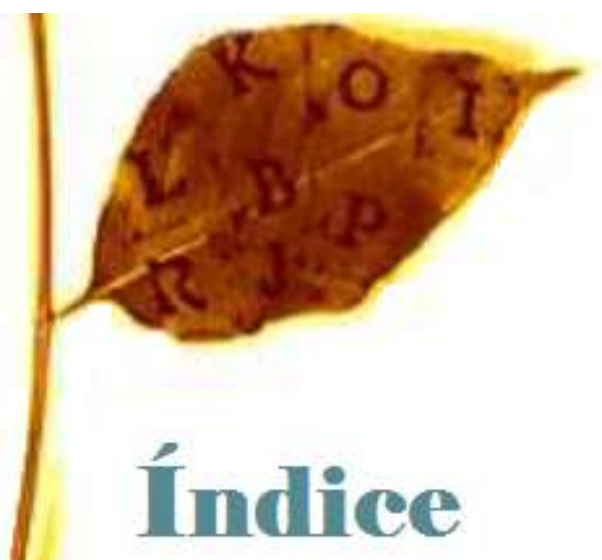

Artículos

113 ¿"El yo propiamente tal” (I. Kant) o "el originario ser sí mismo" (D. Heinrich)? Sobre algunas características del concepto kantiano de autoconciencia

Heiner Klemme

DOI 10.7203/REK.2.2.9068

126 La noción kantiana de verdad transcendental

Stéfano Straulino Torre

DOI 10.7203/REK.2.2.8809

146 Una travesía kantiana a través del Escila constructivista y el Caribdis realista. Apuntes para un abordaje kantiano-constitutivista de las fuentes de la normatividad

Martín Fleitas González.

DOI 10.7203/REK.2.2.8810

174 El malestar kantiano. Filosofía y ciencia al encuentro con la naturaleza Juan Felipe Guevara

DOI 10.7203/REK.2.2.8812

191 ¿Puede haber una fundamentación kantiana de los derechos humanos? Algunas consideraciones críticas

Nuria Sánchez Madrid

DOI 10.7203/REK.2.2.8871

\section{Semblanza}

208 El pensamiento filosófico de Ernesto Mayz Vallenilla

Gustavo Sarmiento

DOI 10.7203/REK.2.2.9021 


\section{Reseñas}

217 Claudia Jáuregi, Fernando Moledo, Hernán Pringe y Marcos Thisted (eds.) Crítica y Metafísica. Homenaje a Mario Caimi. Studien und Materialen zur Geschichten der Philosophie. Hildesheim, Olms, 2015, 460 pp. ISBN: 978-3-487-15237-0.

Alba Jiménez Rodríguez.

DOI 10.7203/REK.2.2. 8814

227 Fernando Moledo: Los años silenciosos de Kant: Aspectos de la génesis de la Deducción Trascendental en la década de 1770. Seguido de la traducción del Legado de Duisburg (ca. 1775). Buenos Aires, Prometeo, 2014, 192 pp. ISBN: 978-987-574-667-1.

Pablo Moscón DOI 10.7203/REK.2.2. 8830

231 Salvi Turró: Filosofia i Modernitat. La reconstrucció de l'ordre del món. Barcelona, Edicions Universitat de Barcelona, 2016, 228 pp. ISBN: 978-84-475-3966-6.

Pedro Jesús Teruel

DOI 10.7203/REK.2.2. 8887

233 Gabriel Rivero: Zur Bedeutung des Begriffs Ontologie bei Kant. Berlín, Boston, Walter de Gruyter, 2014, 247 pp. ISBN: 978-3-11-034180-5.

Luciana Martínez

DOI 10.7203/REK.2.2.8881

Informe

237 Informe sobre el III Congreso de la Sociedad de Estudios Kantianos en Lengua Española (SEKLE)

Luciana Martínez

DOI 10.7203/REK.2.2.9011

\section{Convocatoria}

242 La recepción de Kant en México

Dulce María Granja Castro; Gustavo Leyva Martínez

Eventos y normas para la publicación

244 Noticias y normas para autores 


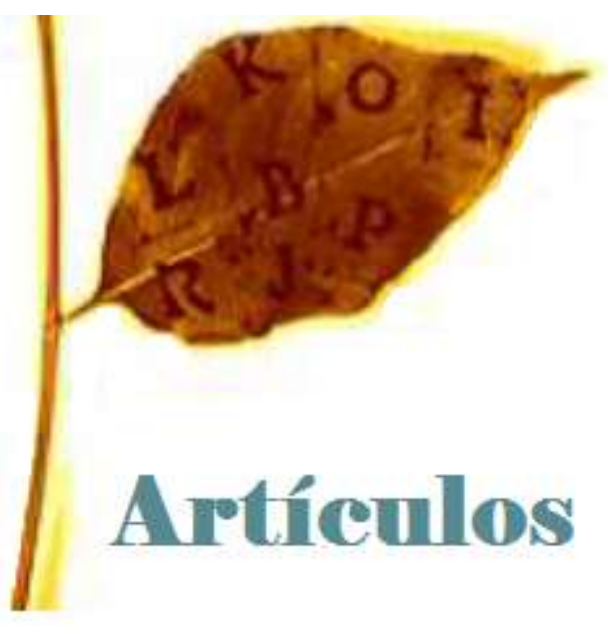




\title{
¿Puede haber una fundamentación kantiana de los derechos humanos? Algunas consideraciones críticas*
}

\author{
NURIA SÁNCHEZ MADRID ${ }^{1}$
}

\begin{abstract}
Resumen
La intención del presente trabajo es indagar si resultad viable considerar al pensamiento de Kant como uno de lo inspiradores del discurso contemporáneo sobre los derechos humanos, con atención a recientes discusiones sobre el tema. En primer lugar, me ocuparé de presentar una lectura de lo que Kant entiende y denomina como «derecho de la humanidad», que debe ponerse necesariamente en relación con la libertad como «único derecho innato», tal y como se formula en la Doctrina del Derecho. En segundo lugar, someteré a debate algunas lecturas recientes del alcance que las tesis kantianas sobre el carácter jurídico del cosmopolitismo y sobre la posesión común originaria de la Tierra podrían tener para una comprensión cabal de lo que cabe identificar en su obra como proto-discusión acerca de lo que hoy en día denominamos derechos humanos. Por último, concluyo que los fundamentos metafísicos que se encuentran a la base de las frecuentes apelaciones kantianas al derecho sacrosanto de los hombres impiden establecer una transición entre el planteamiento de Kant y la concepción radicalmente individualista en que se apoya el discurso de los derechos humanos desde el siglo XX.
\end{abstract}

Palabras clave: cosmopolitismo, derecho, derechos humanos, Kant, libertad, posesión común originaria.

\section{There may be any Kantian Foundation of Human Rights? Some Critical Remarks}

\begin{abstract}
This paper mainly aims at clarifying whether Kant's thought might provide a sound basis for human rights contemporary discourses, focusing on recent discussions about this issue. First I shall attempt to display the meaning of Kant's expression "right of humanity", which should be linked to freedom as the "unique innate right" according to the Doctrine of Right. Second I will discuss some recent readings about the scope of Kant's claims regarding the juridical character of cosmopolitanism and the original common possession of the earth, which could justify to see Kant as a proto-founder of what we understand as human rights in our times. Third I will claim that the metaphysical groundings of Kant's usual hints to the sacred right of humanity prevent to argue for a transition between his argumentation and the clearly individual agent-based conception of human rights from the XXth Century.
\end{abstract}

Key words: Cosmopolitanism, Freedom, Human Rights, Kant, Original Common Possession, Right.

La intención principal del presente trabajo es proporcionar argumentos en contra de la viabilidad de considerar el pensamiento de Kant como uno de los inspiradores del discurso contemporáneo sobre los derechos humanos, con atención a recientes discusiones sobre el tema, en las que destacaremos especialmente algunas perspectivas críticas (O’Neill, Flikschuh y Sangiovanni) y dos aproximaciones más optimistas (Huber y Pinheiro Walla) acerca de la posibilidad de considerar a Kant un protofundador de la teorización de tales derechos básicos. Se trata de una de las cuestiones de mayor

* Este trabajo procede de una investigación resultante de los Proyectos Naturaleza humana y comunidad (III). ¿Actualidad del humanismo e inactualidad del hombre? (FFI2013-46815-P) y Retóricas del Clasicismo. Los puntos de vista (contextos, premisas, mentalidades) (FFI2013-41410-P), concedidos ambos por el Ministerio de Cultura del Gobierno de España. Agradezco a la Universidad Complutense la concesión de una licencia de estudios en julio de 2016, que me ha permitido elaborar el presente trabajo en el Kant Archiv de la Universidad Philipps de Marburgo. También estoy en deuda con los profesores Gerhard Schröder y Werner Stark por su generosidad intelectual y calurosa recepción en aquella universidad, tan ligada al estudio de la obra de Kant.

${ }^{1}$ Universidad Complutense de Madrid. 
actualidad de la obra de Kant y de uno de los puntos más directamente relacionados con la discusión contemporánea sobre las bases y alcance de la justicia global, que autores como Pogge han remitido sin reticencias a Kant como uno de sus referentes clásicos, especialmente al hilo de su ya dilatada polémica con Rawls ${ }^{2}$. Mi propuesta de lectura estará articulada en torno a los siguientes apartados. En primer lugar, me ocuparé de presentar una lectura de lo que Kant entiende y denomina como 'derecho de la humanidad', que debe ponerse necesariamente en relación con la libertad como 'único derecho innato' según la Doctrina del Derecho. Intentaré fundamentar mi valoración positiva de propuestas recientes realizadas por algunos de los especialistas mencionados para considerar tal mención del derecho en Kant como una idea trascendente, que estaría más destinada a orientar el juicio de los gobernantes que a imponer a los representantes legítimos de la soberanía estatal tareas y fines concretos. En segundo lugar, someteré a discusión algunas lecturas recientes del alcance que las tesis kantianas sobre el carácter jurídico del cosmopolitismo y sobre la posesión común originaria de la Tierra podrían tener para una comprensión cabal de lo que cabe identificar en su obra como protodiscusión acerca de lo que hoy en día denominamos derechos humanos. Por último, intentaré sostener que los fundamentos metafísicos que se encuentran a la base de las frecuentes apelaciones kantianas al derecho sacrosanto de los hombres impide establecer una armoniosa transición entre la postura de Kant y la concepción radicalmente individualista en que se apoya el discurso de los derechos humanos en el siglo XX. En este sentido, quizás con excepción de Hannah Arendt (1949, 1951/2006), no podría afirmarse sin caer en un arriesgado anacronismo un solapamiento fructífero de intereses entre las afirmaciones de Kant y las realizadas por autores como Beitz (2003, 2009) o Tasioulas (2010), lo que a mi entender rebaja considerablemente el optimismo que la investigación sobre justicia global ha mostrado a menudo ante al cuerpo doctrinal suministrado por uno de los pensadores clave de la Ilustración europea ${ }^{3}$. Será recomendable tener en cuenta, en un planteamiento como el que pretendemos abordar, la observación siguiente de Kleingeld sobre el uso plural del término cosmopolitismo en Kant:

En la discusión intelectual de finales del siglo XVIII la terminología del 'cosmopolitismo' (y expresiones relacionadas) tenía un sentido coloquial y otros más específicos, y todos están representados en la obra de Kant, que usa el término 'ciudadano del mundo' coloquialmente para referir a una persona de mente amplia (no cerrada) y benevolente, 'amiga de la humanidad' (V-Mo/Phil, AA 2:448; V-Mo/Mrong, AA 27:1550). Pero también usa el lenguaje del cosmopolitismo en un sentido más específico. Le confiere un significado literal en el contexto de su filosofía legal y política, pero lo usa metafóricamente en su filosofía moral (Kleingeld 2016: 14).

Participo de las sospechas que algunos especialistas han expresado a propósito de la facilidad con que teóricos del cosmopolitismo vinculado a las teorías sobre la justicia global consideran a Kant una fuente inspiradora de sus propios discursos ${ }^{4}$, aunque por supuesto no querría silenciar en modo alguno que esa misma línea de investigación cuenta con teóricos que no perciben como algo necesario la referencia a este autor. Considero que esta tendencia constatable

\footnotetext{
${ }^{2}$ Vid. Pogge (2014: xviii): “Kant ha pensado intensamente problemas (...) referidos a los medios por los que puede alcanzarse una sociedad justa a partir de un status quo imperfecto, al modo en que el progreso moral ha aparecido y puede hacerlo en la historia y a la interacción entre progreso e instituciones sociales y progreso de las disposiciones morales". Todas las traducciones al español de los pasajes citados son mías.

${ }^{3}$ Sobre la crítica de este optimismo - e incluso entusiasmo, desde la crítica de las posiciones de Applbaum- puede acudirse a Flikschuh (2016: 282-283): "Buena parte del pensamiento cosmopolita actual parece contener elevadas cargas de emocionalidad. Hasta cierto punto es comprensible: los retos morales son elevados y la necesidad de acción, urgente. Muchos pensadores actuales del cosmopolitismo se consideran inspirados por Kant; Applbaum entre ellos. (...) A este propósito, las observaciones dispersas por Kant en la Doctrina del Derecho sirven de antídoto crítico. Mientras que los primeros escritos cosmopolitas de Kant con frecuencia dejan entrever el fervor moral en cierto modo reflejado en la mayor parte de la teorización cosmopolita actual, la distinción del último texto de Kant entre tres formas interdependientes de derecho público esboza un marco institucional posible para el pensamiento cosmopolita, que puede funcionar como contrapeso de formas excesivamente entusiastas de cosmopolitismo". ${ }_{4}^{4}$ Me refiero por ejemplo al comentario siguiente de Cavallar (2015: 165): "Incluso si Kant es habitualmente la referencia clave del nuevo cosmopolitismo, permanece con frecuencia confuso cuál ha sido su contribución o qué es lo que defiende. Hay a menudo referencias a "la perspectiva kantiana", "el legado kantiano", "la tradición kantiana" o al cosmopolitismo "en sentido kantiano", pero no hay consenso acerca de lo que estas expresiones implican".
} 
bibliográficamente no suministra pruebas suficientes que acrediten la legitimidad de hacer de Kant un fundamento evidente de tales teorías contemporáneas del cosmopolitismo ${ }^{5}$. Más bien, sería deseable reconocer que la consideración de que los escritos de Kant hayan podido inspirar teorías actuales sobre la dignidad y la protección de los seres humanos a escala global ha desembocado en algunos errores metodológicos importantes, que como señalaba antes, lindan con la práctica del anacronismo en el análisis conceptual. ${ }^{6}$ Con el propósito de evitar tales problemas será de utilidad tomar también en consideración el siguiente pasaje de Cavallar:

Kant no preconiza un sistema internacional que discrimine frente a estados no republicanos o no liberales, puesto que subscribe el principio de autodeterminación política. Declara la no intervención como una norma básica de la ley internacional, al postular que los Estados que establecen condiciones legales deben ser considerados personas jurídicas (Cavallar 2015: 174).

El pasaje apunta a una cuestión esencial para proceder a una comprensión cabal de la idea kantiana de derecho cosmopolita -elemento de diálogo fundamental entre Kant y la tradición teórica de los derechos humanos-, toda vez que recuerda la aporía que Flikschuh calificara en un trabajo publicado en 2010 como 'el dilema de la soberanía de Kant', consistente básicamente en el desequilibrio que la doctrina kantiana del derecho establece entre el reconocimiento de legitimidad que concede a la forma estado y el margen de agencia política que cabe asignar a instituciones de carácter internacional. Conceder al Estado republicano, es decir, al Estado de derecho en su acepción kantiana, un estatuto dotado de tamaña centralidad en el marco jurídico-político, obliga a discrepar de posiciones que proclaman la viabilidad de una defensa de la intervención de unos Estados en la política interna de otras naciones con fines civilizatorios, defendida por Hruschka y Byrd en su célebre comentario de la Doctrina del derecho ${ }^{7}$ y en varios artículos por Bernstein (2014: 244). ${ }^{8} \mathrm{Se}$ trata de una tesis que, por otra parte, puede considerarse inspirada por Kant, pero no representada, sino en todo caso abiertamente condenada por sus textos, como es el caso del artículo preliminar quinto de La paz perpetua, que conduciría a los defensores de la vigencia del derecho internacional esbozado por Kant a uno de los principales cul-de-sac a los que se ven abocados, a saber, la inexistencia en Kant de un proyecto de extensión cosmopolita del orden republicano hasta convertirlo en orden mundial ${ }^{9}$. Por el contrario, en los textos de las obras a que nos referimos se señalan instrumentos que dejan insatisfechos a tales intérpretes, por su carácter regulativo y no constitutivo, como la emulación y el ejemplo, esto es, medios vinculados a la educación, que a ojos de Kant pueden conducir a hacer de la experiencia revolucionaria francesa una ocasión para la reforma política y el progreso civilizatorio en el resto de naciones, como veremos un poco más adelante. A falta de instrumentos coactivos, que estima ilegítimos racionalmente, Kant muestra su confianza en que la mímesis cuente con cierta eficacia en el ámbito de las relaciones internacionales entre los

\footnotetext{
${ }^{5}$ Junto con la reciente monografía de Cavallar (2015), la dedicada por Kleingeld (2011) a la procedencia histórica y justificación del cosmopolitismo en Kant sigue siendo la referencia clave para cualquier estudio sistemático sobre este tema.

${ }^{6}$ En este sentido estamos en deuda con el valiente estudio de Oliver Sensen (2011), que ha supuesto un auténtico punto de inflexión en el estudio de la razón práctica kantiana a nivel internacional y ha recibido sugerentes objeciones en la sección "Berichte und Diskussionen" del volumen 106/1 (2015) de la revista Kant Studien.

${ }^{7}$ Nos parece problemático el siguiente comentario de Byrd/Hruschka (2010: 195), pues parece extender la coacción autorizada por el postulado del derecho público en la Doctrina del derecho $(\$ 42)$ a las relaciones entre distintos Estados o entre Estados constituidos y naciones sin Estado: "Kant sostiene que los Estados tienen un derecho en el estado de naturaleza a obligar coactivamente a las naciones vecinas a ingresar en un Estado de Estados jurídico. Si sus vecinos no quieren ingresar en un estado jurídico, el Estado puede declarar la guerra para obligar coactivamente a sus vecinos a hacerlo. Una guerra declarada para "establecer un Estado que aproxime al estado jurídico" (MS, RL, § 55, AA 06: 344) debe permitirse, toda vez que se exige a los Estados abandonar el estado de naturaleza e ingresar en un estado jurídico".

${ }^{8}$ Bernstein sostiene en este artículo una posición más matizada que en su trabajo anterior (2008: 93). Vid. también Tasioulas (2010).

${ }^{9}$ Sobre estas dificultades para rastrear en Kant un derecho a la intervención militar con fines civilizatorios, que Kant tanto tacha como uno de los peores vicios de la humanidad en su crítica al colonialismo, puede acudirse a mis trabajos (Sánchez Madrid 2015b: 51-55, 2016). Una crítica de la convicción kantiana de que la soberanía estatal es indivisible, por lo que no puede compartirse ni distribuirse entre Estados -tampoco con fines civilizatorios y de aproximación a un estado jurídico público a nivel global-, se encuentra en Pogge (2005).
} 
Estados $^{10}$, funcionando así de modo similar al obstáculo interno que la hipocresía impone a tantos gobernantes con respecto a la transgresión de los principios del derecho (ZeF AA 08: 375-376). Así pues, con arreglo a los textos ante la percepción del sufrimiento de otros pueblos los Estados republicanos pueden ejercer todas las presiones posibles, con excepción de la acción militar. La reticencia de Kant a reconocer a un Estado como enemigo injusto en la Doctrina del Derecho es muy sintomática a este respecto. Allí afirma que, aunque la sistemática violación de los pactos públicos por parte de un Estado pusiera en peligro la libertad de los demás, de suerte que estos se vieran obligados a unirse y arrebatarle el poder, ello nunca podría acontecer con la intención de "repartirse el país” (MS, RL, § 60, AA 06: 349), pues ello significaría arrebatar a un pueblo su legítimo derecho a dotarse a sí mismo de una constitución.

El estatismo en torno al que se construye el cuerpo doctrinal de la teoría racional del derecho ha sido matizado por otros reconocidos especialistas en el pensamiento político-jurídico de Kant -como Williams-, singularizando el punto de vista de la vertiente cosmopolita que atraviesa ese mismo pensamiento, hasta el punto de referirse a la viabilidad de un "régimen legal internacional" (Williams 2014: 24), promovido por la presencia en Kant de una multiplicidad de niveles en el planteamiento de la ley y los derechos. Ahora bien, a nuestro juicio debería subrayarse suficientemente el hecho de que todas las exhortaciones a la extensión de la legalidad republicana y sus mecanismos de garantía a la totalidad del planeta no pueden servirse del recurso a la coacción (vid. Sánchez Madrid 2015a: 60-65). En el trabajo anteriormente citado el propio Williams alude justamente a las ventajas derivadas de la clara conexión entre consenso y derecho en la teoría kantiana de la federación, toda vez que confirma que las naciones que se avienen a conformar una entidad internacional dotada de autoridad política han alcanzado una plena madurez política, mientras que las que deben ser aún interpeladas y exhortadas a hacerlo demuestran con su conducta no estar "preparadas para un conjunto de relaciones completamente legales con otros estados" (Williams 2014: 25). Kleingeld, por su parte, ha situado -con mayor acierto que en el caso de Williams a nuestro entender- en el concepto de patriotismo el gozne de efectiva unión entre el derecho político estatal y el derecho cosmopolita, sosteniendo que la culminación de la constitución republicana se encuentra vinculada en Kant con la ampliación de los límites del derecho y la progresiva conversión de todos "los hijos de la Tierra" en ciudadanos del mundo" ${ }^{11}$ La línea argumentativa del presente trabajo comparte con esta destacada intérprete que la madurez estatal es la principal evidencia reconocida por Kant que nos permite confiar en que la unión pacífica cosmopolita sea el único destino posible para los distintos pueblos ${ }^{12}$. Ahora bien, el camino hacia ese estado de madurez no puede protagonizarlo nadie más que el pueblo que debe transitarlo, sin que el derecho cosmopolita o la invocación de unos discutibles 'derechos humanos' puedan poner en solfa esta profunda convicción kantiana.

\section{Una visión trascendente de los derechos humanos}

\footnotetext{
${ }^{10}$ Véase la lúcida observación Sangiovanni sobre este punto (2015: 679): “[e]l derecho innato y sus corolarios no pueden suministrar la base para un instrumento con autoridad regional en el ámbito de los derechos humanos. Como mucho, si queremos permanecer dentro del marco kantiano, podríamos considerar un cuerpo internacional que proporcione meras opiniones cuyo objetivo fuera asesorar. Tal cuerpo podría emitir recomendaciones a los Estados sobre cómo mejorar su protección del derecho innato (y el sistema recíproco de libertad que manda tal derecho), pero esto no podría imponer obligaciones genuinas ni exigir coactivamente el cumplimiento de sus juicios de ninguna manera. Esto queda muy lejos de los tipos de derechos humanos por los que nuestros contemporáneos abogan y por los que los expertos consideran estar luchando".

${ }^{11}$ Vid. Kleingeld (2016: 19): "Kant sostiene que los deberes cosmopolitas y patrióticos son compatibles, por cuanto ambos están orientados a la realización del derecho. Pues si la república es ella misma requerida como parte de la empresa de producir una 'condición cosmopolita' del derecho, cumplir con el deber propio como ciudadano de una república está en la misma línea que el deber cosmopolita que le compete (Kleingeld 2000). Al basarse este argumento en la noción de república, no implica que los sujetos de un estado 'despótico' tengan un deber patriótico similar".

${ }^{12}$ Merece la pena profundizar en la crítica de las prácticas coloniales elaborada por Kant y su proyección en la actualidad, de lo que se han ocupado Ajei/Flikschuh (2014: 223): "Kant puede haber estado en condiciones de reconocer el -entonces incipienteproblema de la mentalidad colonialista. Tenía los recursos teóricos para ello. Tales recursos remiten al formalismo de Kant, más específicamente, a su formalismo jurídico".
} 
Un número relevante de afirmaciones de Kant sobre la condición cosmopolita de los Estados ofrecen un aspecto inequívocamente conservador, si las comparamos con las expectativas de especialistas en justicia global como Pogge, Risse o Ronzoni ${ }^{13}$, toda vez que juicios y consideraciones políticas palmariamente razonables $-\mathrm{y}$ con toda probabilidad indiscutibles para cualquier ciudadano de una democracia deliberativa actual, no estarían en condiciones de vulnerar ni un ápice la autonomía política de los Estados, sancionada en la Doctrina del derecho- ${ }^{14}$ Como si de la contracara de semejante parecer se tratase, es cierto que Kant pondera, como recordábamos antes, la capacidad de una joven república europea -la derivada de la Revolución francesa- como un foco en condiciones de impulsar el progreso de la Ilustración en la historia humana. Pero la fuerza del argumento estriba en la facultad que se adscribe a tal república para actuar como modelo a emular por otras naciones, que advertirán paulatinamente la necesidad de su coordinación federal con vistas a suprimir la guerra, fuente principal del mal en la historia:

Nosotros podemos hacernos una idea de cómo se puede realizar esta idea de una federación, podemos imaginarnos su realidad objetiva, que ha de extenderse paulatinamente a todos los Estados y conducir así a la paz perpetua: si la fortuna dispone que un pueblo fuerte e ilustrado se transforme en una república (la cual debe tender, por su propia naturaleza, a la paz perpetua), esa república puede convertirse en el núcleo de la unión federativa con otros Estados, que se unirían a ella, garantizando así un estado de libertad de conformidad con la idea de derecho internacional y que se iría ampliando poco a poco con más adhesiones de este tipo (ZeF AA 08: 356$)^{15}$.

Esta opción por la emulación como una de las puntas de lanza del derecho cosmopolita kantiano dificulta asimismo fundamentar deberes jurídicos destinados a proteger la agencia individual alrededor del mundo, toda vez que son los Estados los que, distributiva y no colectivamente, están llamados a ofrecer a los sujetos la promoción al estatuto de ciudadano, esto es, a volver efectiva su libertad como único derecho innato. Y la constitución de una autoridad política estatal impide a los agentes individuales realizar una suerte de revisión regular de la legitimidad de sus respectivos Estados, que pudiera justificar un presunto derecho de resistencia a aquella suprema autoridad político-jurídica. Por el contrario, semejante itinerario incitador a la rebeldía frente a la única autoridad pública legítima debería declararse sencillamente como fuera de la ley. Todo en la argumentación de Kant está articulado de manera que el poder se destruya a sí mismo en caso de que no se respete la consistencia interna de la comunidad política ${ }^{16}$, que, a diferencia de lo que caracteriza a una sociedad, procede de una subordinación sin paliativos de los ciudadanos al legislador. De acuerdo con mi lectura de la célebre exhortación de Kant al hecho de que la injusticia surgida en un rincón de la tierra no puede sino sentirse en cualquier otro (ZeF AA 08: 360), la condición básica para su interpretación cabal no pasa por ver en ella la apelación a fundar una unión política internacional, destinada a proteger a todos los seres humanos que habitan la tierra. Por el contrario, tiendo a pensar que los Estados son a juicio de Kant el único espacio político posible en el que la violación del derecho podría sentirse y recibir consiguientemente una contundente réplica. Todo parece indicar en

\footnotetext{
${ }^{13}$ Sobre la dificultad para cohonestar las líneas de argumentación de estos autores con los escritos kantianos, puede acudirse a mi trabajo (Sánchez Madrid 2014).

${ }^{14}$ MS, RL, AA 06: 320: "La razón por la que el pueblo debe soportar, a pesar de todo, un abuso del poder supremo, incluso un abuso considerado como intolerable, es que su resistencia a la legislación suprema misma ha de concebirse como contraria a la ley, incluso como destructora de la constitución legal en su totalidad. Porque para estar capacitado para ello tendría que haber una ley pública que autorizara esta resistencia del pueblo; es decir, que la legislación suprema contendría en sí misma la determinación de no ser la suprema y de convertir al pueblo como súbdito, en uno y el mismo juicio, en soberano de aquel al que está sometido, lo cual es contradictorio". Traducción de J. Conill y A. Cortina. Las obras de Kant aparecen citadas según la edición de la Academia de las Ciencias de Berlín, con arreglo a las indicaciones de la Kant Forschungsstelle de la Univ. de Mainz: http://www.kantgesellschaft.de/de/ks/HinweiseAutorenSiglen_neu.pdf

$\frac{\text { gesellschaft.de/de/ks/HinweiseAut }}{{ }^{15} \text { Traducción de Joaquín Abellán. }}$

${ }^{16}$ Cf. la visión negativa de la 'consistencia interna' de la noción kantiana de soberanía política en Meckstroth (2013: 37): "[i]ncluso en el caso doméstico, son necesarios juicios empíricos entre potenciales candidatos a la soberanía de un territorio y de unos ciudadanos dados. Sin embargo, Kant no proporciona nunca una explicación por principios del modo en que tales juicios puedan defenderse o de quién tenga el derecho a hacerlos en casos de conflicto político".
} 
los textos - podemos restringir nuestra mirada a La paz perpetua- que los Estados dotados de una genuina constitución republicana producen su propia comunidad global, en el bien entendido de que el elemento que fomenta tal federación no es externo, sino interno a la unión civil constituida por cada pueblo o nación, como ocurre en la formación de las galaxias en el cosmos. ${ }^{17}$ Es más, la comunidad cosmopolita está supeditada en Kant a la promoción de la agencia política estatal, que confirma que la comunidad ideal funciona aquí más como un objetivo ideal que como una efectiva autoridad política supraestatal.

Así pues, la teoría kantiana del Estado de Derecho asigna un alcance regulativo a la unión federal, que está llamada a fortalecer las condiciones pacíficas en la Tierra. Comparto en este sentido con Flikschuh su impresión de que nada en la Doctrina del Derecho exige fundamentar derechos humanos específicos, lo que sin embargo no implica la exclusión como principio reflexivo y regulativo de todo principio similar al menos a lo que hoy en día consideramos como derechos humanos. ${ }^{18}$ En efecto, las alusiones habituales en Kant a los derechos de la humanidad como un asunto sagrado recuerda al comandante supremo de una nación que debe cumplir con un compromiso elevado, hasta el punto de resultar coherente considerarlo como una tarea directamente concedida al gobernante por el mismo Dios. El siguiente pasaje de una nota a veces postergada de La paz perpetua puede servirnos de botón de muestra de este parecer:

Los elevados tratamientos que se han dado a los gobernantes han sido tachados de vulgar adulación («ungido de Dios», «administrador de la voluntad divina en la tierra» y «representante de Dios»), pero me parece que estos reproches no tienen fundamento. -Es una gran equivocación decir que este tratamiento haga al gobernante arrogante, pues más bien debe abatirle su espíritu, si tiene entendimiento - algo que hay que presuponer- y piensa que ha recibido un cargo demasiado grande para un hombre, concretamente administrar lo más sagrado que tiene Dios sobre la tierra, el derecho de los hombres, y que tiene que estar constantemente preocupado por haberse situado demasiado cerca del ojo de Dios (ZeF AA 08: 353 , nota) ${ }^{19}$.

El pasaje recuerda también la preocupación manifestada por Kant en la proposición VI de Idea de una historia desde un punto de vista cosmopolita, a propósito de la dificultad que entrañan tanto el arte de gobierno como el de educar, desde el momento que es siempre un ser humano el que ejerce de jefe de otro ser humano, sin poder apelar a una hechura más elevada que invite a otorgarle el mandato. Se trata asimismo de un pasaje útil para distinguir entre una constitución republicana y la democrática, donde la última carece -como es bien sabido- de instrumentos de representación. El recordatorio teológico de Kant desempeña sin duda alguna una función metafórica, y, por tanto, retórica -como las menciones a la Providencia de la Naturaleza en este mismo escrito kantiano-, pero en su retoricidad no deja de señalar que en la tarea de representación política hay siempre un exceso que no puede rebajarse totalmente ni traducirse a términos mecánicos e inmanentes. Un exceso sin el que la teoría kantiana de la política podría reducirse a un modelo mecánico como el de Hobbes. En todo caso, no se abre con ello una vía de trabajo que pueda arrojar esperanzas a los interesados en poner a Kant en diálogo con las preocupaciones de los teóricos de los derechos humanos en la

\footnotetext{
${ }^{17}$ Una imagen que Kant maneja metafóricamente en su obra jurídico-política como suma expresión de organicidad y autonomía La obra primordial para reconocer el alcance político de tal imagen es Historia general de la naturaleza y teoría del cielo (1755). Vid. Sánchez Madrid (2009).

${ }^{18}$ Flikschuh (2015: 665-666): "Diría que nada de lo afirmado en la Doctrina del derecho requiere recurrir al concepto de derechos humanos como fundamento necesario para la reivindicación práctica de una ley positiva. El concepto de derechos humanos es según mi lectura, diferente del de derecho innato, que es indispensable para la justificación moral de las exigencias de propiedad sustantivas de las personas. Por otra parte, concedo que no hay tampoco nada en la Doctrina del derecho que excluya concebir la idea de los derechos humanos como un concepto trascendente relativo al dominio de la elaboración de leyes positivas. En particular, en la medida en que la idea no sea tratada como suministradora de una premisa fundante para legitimar moralmente cierta legislación, nada habla en contra de su posible estatus como una idea reflexiva subjetivamente necesaria que surge del mismo proceso de producción de leyes públicas".

${ }^{19}$ Vid. también O’Neill (2005) y Dorsey (2005).
} 
actualidad. Frente a tales expectativas, mi propuesta sería tomar en consideración, a partir fundamentalmente de la lectura de Flikschuh, las siguientes tres tesis:

i) La teoría kantiana del Estado de derecho no desemboca en un conflicto de legitimidad entre la autoridad estatal y la federal.

ii) La apelación a los derechos de los seres humanos coincide con el punto de vista que Dios o la Providencia dirigen a los asuntos humanos.

iii) No hay rastro en el pensamiento de Kant de una concepción en clave subjetiva de los derechos humanos, en tanto que derechos asignados a los agentes individuales por encima de su pertenencia a un Estado.

A mi juicio, el tercer punto es la clave para comprender debidamente los dos primeros, dado que explica por qué Kant no considera la agencia individual, sino más bien al punto de vista de una divinidad de corte deísta encarnada en la Providencia como Sabiduría suprema, como instancia suprema desde la que plantear la cuestión de los derechos de la humanidad. La referencia explícita a esta clase de derechos puede rastrearse en escritos de Kant como La paz perpetua y Teoría y práctica. Aparte del pasaje reproducido de La paz perpetua, el trabajo preparatorio de este mismo escrito sostiene, por ejemplo, que la constitución republicana se caracteriza por su correspondencia con el "derecho de la humanidad" (VAZeF AA 23: 164), es decir, por su cumplimiento de los principios de libertad e igualdad y su disparidad con respecto a la configuración despótica del Estado. Asimismo, La paz perpetua recoge en la parte final de su tercer artículo definitivo la consideración del derecho cosmopolita como una parte constituyente del sistema del derecho público, concretamente en los términos del "complemento necesario del código no escrito del derecho político y del derecho internacional para un derecho político de los hombres como tales" (ZeF 8: 360; trad. de Joaquín Abellán), encargado de actuar de manera positiva en la aproximación a una pacificación progresiva de los distintos Estados. Finalmente, el escrito sobre el Gemeinspruch sostiene que de la confianza en un progreso constante del género humano hacia lo mejor depende la victoria sobre "una premeditada violación recíproca de los más sacrosantos derechos del hombres" (TP AA 08: 307; trad. de Roberto R. Aramayo). Pero ¿cuál es el lugar teórico al que remiten todas estas alusiones a un derecho de los hombres que ningún interés parcial, por legítimo que sea, puede transgredir? La perspectiva interpretativa adoptada por autores como Onora O’Neill, Katrin Flikshuh y, con una radicalidad que no puedo compartir, Andrea Sangiovanni, son de ayuda -como esperamos haber mostrado- para abordar esta primera tarea, optando por la hermenéutica de la expresión como una suerte de corolario trascendente de la hechura racional del derecho en Kant, cuyo baricentro es indefectiblemente estatal.

Como intentaré probar en el segundo apartado, Kant muestra en su teoría del derecho tener una conciencia clara acerca de las consecuencias que la posesión común originaria de la Tierra y la esfericidad de nuestro planeta generan en el plano jurídico, de suerte que este hecho empírico se vea dotado de una función política regulativa. Pero hay otro elemento esencial para sostener que en Kant la protección de cualquier vida humana sobre la Tierra compete a la autoridad estatal. Nos referimos a la necesidad de que la protección de los sujetos y, por tanto, el respeto de su derecho innato a la libertad, se corresponda con un control suficiente por parte de la autoridad pública de los recursos materiales y del territorio del que se trate en cada caso. Se apunta con ello al frecuente reproche a los derechos humanos -véase Arendt (1949, 1951/2006)- por su abstracción con respecto a las condiciones fácticas concretas sin las que no pueden considerarse dotados de eficacia legal. La siguiente observación de Flikschuh me parece sumamente dilucidadora con respecto al vínculo kantiano entre las condiciones materiales de la soberanía estatal y el respeto efectivo de esos derechos: 
No tengo conocimiento de que la mayor parte de los estados establecidos hayan llegado de manera característica a la garantía de la provisión de servicios centrales público -saneamiento, educación, servicios de salud- sobre la base de compromisos explícitos con los derechos humanos. Por el contrario, la preocupación sería por lo habitual mejorar la capacidad soberana, esto es, garantizar el ejercicio efectivo y legítimo de la autoridad coactiva a través de la asunción parcial de responsabilidad pública. (...) Así pues, hasta que no reconozcamos un vínculo entre el cumplimiento de los derechos humanos y la competencia soberana, por un lado, y entre la competencia soberana y el suministro de bienes públicos, por el otro, puede haber razón para pensar que la mejor ruta hacia los derechos humanos consista en el fortalecimiento de la competencia soberana, especialmente en el nivel del suministro de bienes públicos (Flikschuh 2011: 34-35) ${ }^{20}$.

El texto no tiene el propósito de justificar la corrupción de tantos sedicentes Estados de derecho en tantas regiones de la Tierra, sino más el de enfocar algunas dificultades que la mayor parte de los Estados postcoloniales encuentran cuando pretenden desempeñar una verdadera agencia política. Flikschuh, como anteriormente O'Neill (2005), anima a los intérpretes del cosmopolitismo kantiano, entre los que se encuentra, a no olvidar el nexo existente entre la autoridad estatal y el compromiso con la protección política efectiva de un territorio y una sociedad, tarea que comporta el control de los recursos naturales y manufacturas del país. Por el contrario, la historia transmite que el control que algunos gobiernos tienen sobre sus propios bienes y recursos es realmente escaso, hecho que necesariamente debe ser tomado en consideración en una lectura de la actualidad de la teoría kantiana del Estado de derecho, es decir, de su modelo republicano.

2. El alcance de la posesión común originaria de la Tierra para una fundamentación de los derechos humanos en clave kantiana

A pesar de que mi posición ante el uso habitual que se realiza de los textos de Kant en buena parte de las discusiones actuales sobre los derechos humanos es escéptica, me parece asimismo desenfocado optar - como es el caso de Sangiovanni- por el camino radicalmente contrario al que venimos de criticar. A mi juicio, no sería sostenible desconectar enteramente la fórmula de la humanidad, tal y como queda expresada en la Fundamentación, el concepto kantiano de dignidad y el principio universal del derecho ${ }^{21}$. Por el contrario, considero que debe destacarse la presencia en la Doctrina del derecho de pasajes clave acerca de un derecho universal de los seres humanos a desplazarse libremente por la tierra, imposible de fundamentar sin recurrir a conceptos tales como el de dignidad humana y a la consideración del ser humano como un fin en sí mismo en virtud de su libertad. En esta línea, cabría sostener que la posesión común originaria de la Tierra que todos los seres humanos comparten de manera indefectible será esencial para visibilizar el nivel de lo regulativo en el propio cuerpo del derecho racional y calibrar así debidamente la estructura sistemática que lo jurídico posee en Kant:

Todos los hombres están originariamente (es decir, antes de todo acto jurídico del arbitrio) en posesión legítima del suelo, es decir, tienen derecho a existir allí donde la naturaleza o el azar los ha colocado (al margen de su voluntad). Esta posesión (possessio), que difiere de la residencia (sedes) como posesión voluntaria y, por tanto, adquirida y duradera, es una posesión común, dada la unidad de todos los lugares sobre la superficie de la tierra como superficie esférica: porque, si fuera un plano infinito, los hombres podrían diseminarse de tal modo que no llegarían en absoluto a ninguna comunidad entre sí, por tanto, esta no sería una consecuencia necesaria de su existencia sobre la tierra (MS, RL, § 13, AA

${ }^{20}$ Cf. Ajei/Flikshuh 2014: 238-244; O’Neill 2005, passim; Dorsey 2005, passim.

${ }^{21}$ Sangiovanni (2015: 689): "[s]i la concepción de la dignidad de Kant y la Fórmula de la Humanidad no son ni necesarios ni suficientes para reconstruir la teoría del derecho de Kant, entonces no es posible derivar una teoría de auténticos derechos humanos kantianos tomando únicamente esta base. Aunque alguien pueda reinventar el concepto de dignidad de Kant para otros usos, esto le llevaría más allá de lo que una auténtica teoría kantiana podría autorizar”. 
06: 262 , trad. citada).

El pasaje citado enfoca con toda intensidad el alcance que las ideas de la razón, como la de comunidad originaria del suelo, poseen en el interior de la propia teoría jurídica kantiana, que no en vano se reivindica racional y no inspirada por la práctica de los jurisconsultos. Lo que reclama el texto es que ninguna autoridad pública menosprecie ni ponga en riesgo una realidad fáctica que sencillamente no es materia de elección, sino consecuencia de una hechura física, como es el hecho de que todos los seres humanos comparten una misma Tierra de la que no pueden escapar y en la que, llegado el caso, han de poder quedar resguardados de las inclemencias de la naturaleza y de las agresiones de otros individuos. En definitiva, la comunidad originaria de la Tierra comparece en la doctrina racional del derecho como la expresión más acabada de la lex iusti que debe inspirar los esfuerzos de toda sociedad por salir del estado de naturaleza, es decir, de barbarie (MS, RL, AA 06: 236-237). Recientes publicaciones de Pinheiro Walla y de Huber se han ocupado precisamente de esclarecer el estatuto conceptual de afirmaciones de Kant como la anteriormente citada, abriendo nuevas vías de interpretación en clave regulativa del derecho kantiano. Parece fuera de toda duda que Kant alude en pasajes como el precedente a una fuente de normatividad jurídica -o proto-jurídica-, pero la cuestión sería determinar de qué tipo de normatividad debería hablarse a este propósito. Por de pronto, es preciso señalar que el texto sirve de base para que en las consideraciones kantianas sobre el derecho cosmopolita cada sujeto vea reconocido su derecho a visitar -de manera voluntaria, como comerciante, o involuntaria, cuando se trata de un naufragio, por poner dos ejemplos distintosdistintas regiones de la Tierra -naciones o no-, de las que ni es oriundo ni ciudadano. Recordemos que, como es bien sabido, el derecho cosmopolita, una versión del actual derecho internacional privado en definitiva, no reconoce al individuo ningún derecho de residencia en el territorio en que recala, sino más bien un derecho de visita o de auxilio provisional, en el caso de que su voluntad o accidentes naturales le hayan conducido hasta allí. Considero que en este contexto de análisis, Pinheiro Walla ha apuntado muy razonablemente a la conveniencia de considerar el derecho cosmopolita como signo de una normatividad que la lex iusti exige de manera continuada en un mundo dominado por autoridades de nivel estatal:

A pesar de que se trata de un desarrollo positivo en la transición de la lex iusti, a través de la lex iuridica, a la lex iustitiae distributivae en la condición civil, la lex iusti no se vuelve superflua en la condición civil, sino que sigue siendo la fuente de la normatividad y, consiguientemente, de la legitimidad, de los ulteriores desarrollos del derecho. La necesidad de mantener la compatibilidad del desarrollo del derecho con su fuente normativa a priori es lo que origina el derecho cosmopolita. En este sentido, derecho cosmopolita tiene una función en la teoría de Kant similar al derecho de necesidad en Grocio y a los derechos imperfectos en la teoría de Pufendorf (Pinheiro Walla 2016: 17).

Encuentro acertado y participo de la idea de que las tareas y fines impuestos a la humanidad por el respecto de lo recogido bajo la lex iusti no desaparecen con el advenimiento de la condición civil, al no poder solaparse enteramente con la constitución de un Estado republicano. También comparto la propuesta de comparar los principios racionales inspiradores del cosmopolitismo kantiano con lo que teóricos del derecho natural, como Grocio y Pufendorf consideraron derecho de necesidad o derechos imperfectos, cuya miopía con respecto a las bases racionales del derecho, sin embargo, Kant critica con dureza en el curso Naturrecht Feyerabend. Podría decirse que Kant procede a una reformulación en clave inmanente y racional del ius necessitatis de los teóricos del derecho natural. Estamos de acuerdo en la tesis de que los fines de la lex iusti van mucho más allá de los consumados por la lex justitiae distributivae, iluminando el fondo racional que sostiene la entera normatividad jurídica en Kant. En este contexto, y reconociendo el provecho extraído de la apuesta de Pinheiro Walla, mi propuesta sería más bien considerar la alusión de Kant a una posesión común originaria de la Tierra como una tesis de naturaleza pre-jurídica -y pre-política- que impone a los Estados y naciones el respeto de un factum originario que ninguna unión civil estaría legitimada para 
obviar $^{22}$. Sin embargo, no me atrevería con ello a declarar que el valor concedido por Kant a este principio pre-jurídico justifique su consideración como el esbozo de una teoría de los derechos humanos. A pesar de que Pinheiro Walla no desemboca expresamente en tal asociación, sus conclusiones me parecen menos escépticas que las mías en relación con el asunto que nos ocupa. Recuperando la argumentación de Flikschuh acerca de la presencia de estos derechos en Kant como una suerte de resto trascendente para toda tarea legislativa y de gobierno, creo que el principio aludido forma parte de la satisfacción de los fines impuestos por el "único derecho innato" en la Doctrina del derecho (MS, RL, AA: 237-238), pero no se convierte con ello en elemento de diálogo posible con las teorías sobre la justicia global. No me atrevo tampoco a ir tan lejos como Huber en la valoración de tales exigencias racionales en la doctrina racional del derecho, por cuanto ve aceptable una suerte de ampliación de la noción de la política en Kant, en caso de atender debidamente a pasajes como el del citado $\$ 13$ de la Doctrina del derecho:

La concepción kantiana va más allá de la perspectiva "política" al no reducir lo "político" a relaciones verticales entre el gobernante y los gobernados, sino al concebirlo de manera más amplia, describiéndolo como un tipo particular de relación moral entre todos los individuos. El razonamiento defendido es así bidimensional: por un lado, concuerda con las explicaciones ortodoxas de que los derechos humanos son principios horizontales frente a todos los individuos. Sin embargo, la diferencia crucial estriba en que esta exigencia de derechos ya contiene una implicación política, puesto que solo bajo una autoridad política es posible el ejercicio consistente de la capacidad de cada cual de elegir y actuar. Por otro lado, en un nivel vertical (una vez que nos encontramos en la condición política) el derecho innato describe una exigencia válida frente a la autoridad política para ser tratados como iguales ante la ley y, así, constituye un criterio racional para toda posible ley legítima. (...) Lo que Kant capta -y lo que hace de él un verdadero pensador republicano- es que las relaciones "políticas" no se reducen solo a las existentes entre los gobernados y el gobernante. Las relaciones "políticas" son un tipo particular de relaciones morales que tenemos con otros individuos -a saber, unas relaciones que requieren coordinación y arbitraje a través de las autoridades políticas-(vid. Huber 2013: 137, 140).

La propuesta interpretativa de Huber puede resultar sugerente. En efecto, lo es, en la medida en que contribuye -como vimos con Flikschuh y Pinheiro Walla- a destacar la función desempeñada por lo regulativo en la doctrina kantiana del derecho. El cosmopolitismo kantiano comporta una intensa conciencia del alcance metafísico y político del hecho de que el conjunto de los seres humanos compartan un mismo espacio finito. Sin embargo, no me parece que semejante paso pueda conducir fácilmente a una ampliación de la noción que Kant sostiene acerca de la autoridad jurídicopolítica, que los habitantes de la Tierra solo conocerán en caso de convertirse en ciudadanos de un Estado. El proto-derecho reconocido a todo ser humano para tener un lugar que visitar o donde resguardarse allí donde recale en la Tierra no cuenta con el valor de un derecho fundamental que guíe un listado de derechos subsiguiente, esto es, no podemos encajarlo en el principio de división jurídica de la Doctrina del derecho, donde la libertad para coexistir con cualquier otro según una ley universal señala en dirección a la fundación de un Estado republicano, no a una instancia internacional encargada de velar por los derechos del hombre y del ciudadano. Frente a la última expectativa, la adquisición llevada a cabo por cada sujeto encarna y da contenido al proto-derecho mencionado, que no conserva nada más, pero tampoco nada menos, que una función regulativa sobre el derecho público ${ }^{23}$.

${ }^{22}$ Es de interés tener a la vista igualmente la reflexión de Huber (2013: 138): "Cuando hablamos de un "proto"-derecho, debemos tener en cuenta que no se trata del único derecho fundamental (no justificable en sí mismo), que justificaría en ese caso el resto de derechos. Esto conduciría a una suerte de realismo moral con el que la filosofía crítica de Kant tiene problemas. Todo lo que podemos decir es simplemente que tener derecho es una exigencia válida". Sobre esta valoración de la posesión común originaria del suelo véase también Byrd/Hruschka (2010: 128).

${ }^{23}$ Me parece muy acertada la siguiente observación de Pinheiro Walla, crítica con la interpretación de Flikschuh acerca de la relación que mantienen derecho innato y adquirido en Kant. Véase (2016: 177): "[n]o hay por qué pensar, como ha sostenido Flikschuh, que Kant parte "del hecho de la adquisición individual para llegar a la idea de la posesión común originaria" y que, por tanto, ha "invertido" la secuencia de la ley natural desde la posesión común a la adquisición individual. Esto significaría tomar la 
Recordemos a este respecto la insistencia de Kant en que la unión civil no constituye ninguna sociedad, toda vez que el pactum subiectionis es la operación definitoria del derecho sancionado públicamente. La llamada de atención sobre el hecho de que "el soberano (imperans) y el súbdito (subditus) (...) no son compañeros, sino que están subordinados uno a otro, no coordinados" (MS, RL, § 41, AA 06: 306-307) plantea una objeción elocuente a la observación de Huber, según la cual las relaciones políticas no se reducen en Kant a las existentes entre gobernante y gobernados. Precisamente la definición de la unión civil como una estructura jerárquica limita considerablemente las expectativas de ampliar sustancialmente el campo de acción político en términos horizontales.

Mi principal objeción a los planteamientos expuestos radica en que la condición impuesta a los Estados por el derecho cosmopolita, que les arrebata la facultad para coaccionarse recíprocamente, sería el pendant de la dimensión trascendente y regulativa que caracteriza a esta parte del derecho racional. Esto se advierte en la formulación kantiana en términos radicalmente formales de lo que la tradición ortodoxa sobre las presuntas causas y virtudes del colonialismo había reconocido como doux commerce, de manera que, si bien el derecho innato a la libertad no se solapa sin más con el advenimiento de una propiedad privada reconocida públicamente, no por ello el argumento del derecho innato que todo ser humano tiene para visitar el mundo debería traducirse en un presunto derecho de residencia (MS, RL, § 62, AA 06: 353 y ZeF, AA 08: 358). Teniendo en cuenta la literalidad de los pasajes de Kant sobre el hecho de que pertenecemos a una comunidad cosmopolita, considero que este 'punto de vista global' no podría ofrecer bases sólidas para un concepto presuntamente fundado de justicia global, dado que su efecto inmediato parece ser el de recordar a todos los Estados que deben acatar el derecho universal con vistas a sus desplazamientos. Ahora bien, el derecho cosmopolita no exige ninguna retirada del Estado de sus fronteras políticas legítimas, una exigencia que investigadores especializados en justicia global como Ronzoni (2009) han defendido en varias ocasiones. Investigadores como Huber defienden una visión más optimista de la concepción kantiana de la comunidad cosmopolita, pero a mi entender no del todo fiel a la sistemática kantiana. El pasaje siguiente podrá ayudarnos a aclarar este punto:

Pensarse a uno mismo como un habitante de la Tierra es pensarse a sí mismo como participante en una comunidad cosmopolita de individuos, cuyos destinos están, en un sentido importante, inevitablemente unidos entre sí, pero que al mismo tiempo tienen la capacidad de relacionarse críticamente unos a otros y con las instituciones contingentes, fronteras y lealtades que les separan (...) El cosmopolitismo de Kant no es el de unos seres nouménicos unidos en su humanidad compartida, ni el mundo actual de ciudadanos que comparten una política global. Se trata más bien de un cosmopolitismo de los habitantes de la Tierra: agentes racionales dotados de cuerpo en confrontación física directa con otros agentes iguales a ellos, con los que tienen que compartir el planeta que tienen en común. Se trata de un cosmopolitismo que no ofrece en sí mismo una sencilla guía institucional para un mundo justo, pero que con todo ofrece a los agentes una especie de punto de vista global desde el que pensar y actuar (Huber 2016: 24).

A la luz de mi lectura de las obras fuente citadas, Kant no reconoce ningún derecho a encontrarse en un lugar si este no se ve acompañado por la pertenencia a un Estado como miembro activo. Es más, aquel derecho surge como un derecho de movimiento transitorio que refleja la posesión originaria de la Tierra sin desdibujar la gramática estatista de Kant, simplemente porque el primero es regulativo, mientras que la segunda es constituyente de la libertad sagrada de los seres humanos. Como se ha afirmado antes, mi impresión es que los textos de Kant no ofrecen los

comunidad originaria como constituida por hechos dados empíricamente. Kant es suficientemente claro a propósito de que la comunidad originaria es una idea de la razón y no una comunidad que haya sido "instituida" (gestiftete Gemeinschaft). Esta incapacidad para entender el carácter racional (esto es, originario) de la idea de comunidad de la Tierra es precisamente lo que Kant considera un error de la "comunidad primitiva" (uranfängliche Gemeinschaft, communio primaeva) de Grocio y Pufendors. El que Kant tome como punto de partida la teoría de la ley natural no consiste en una "inversión" de la secuencia de ideas, sino en una redefinición de conceptos centrales de la ley natural en términos de libertad externa". 
resultados deseados por los teóricos de la justicia global y cierta parte de la investigación actual en Kant. Naturalmente, esto no significa que nuestro autor no haya considerado suficientemente las consecuencias procedentes de la comunidad espacial que los seres humanos despliegan sobre el mundo, sino más bien que esta explicación forma parte más del ámbito regulativo de las ideas racionales que de las dimensiones constituyentes del Estado de derecho. En definitiva, los principios regulativos y trascendentales carecen en Kant de capacidad para coaccionar ninguna acción, lo que no significa -como implica su propia denominación- que no deban orientar de manera productiva la agenda de los respectivos Estados.

\section{Conclusión}

La aproximación propuesta a la cuestión de los derechos humanos y la consideración de Kant como uno de sus inspirados clásicos ha pretendido adoptar un tono intermedio en una discusión en la que suele predominar la radicalidad de las posiciones. No pretendo sostener que el valor interno de la humanidad no juegue papel alguno en la obra política de Kant, sino más bien señalar que precisamente los derechos de la humanidad, tan apreciados por este pensador, no tienen un carácter subjetivo y, además, encuentran su principal paladín en la autoridad estatal. En esta secuencia de razones, la alusión kantiana en la Doctrina del derecho a la posesión común que todos los seres humanos tienen sobre la Tierra parece un resultado demasiado pobre a los ojos de los discursos contemporáneos sobre la justicia global y la defensa a esa escala de los derechos básicos de todos los seres humanos. El 'derecho a la membresía' acuñado por Benhabib (2008), heredero del 'derecho a tener derechos' de Arendt, no parece aceptable por el cuerpo doctrinal levantado por Kant a propósito de su teoría de la justicia, toda vez que se mantiene por debajo de la línea de flotación de la pertenencia ciudadana a un Estado. Kant percibe a los mismos visitantes a los que imagina accediendo a otras tierras de las que no son oriundos como ciudadanos de Estados a los que representan, ya sea desde un punto de vista político o como agentes económicos. La otra posibilidad que explicaría la migración la referimos antes, a saber, los incidentes como el naufragio que obliga a recalar en un territorio extranjero.

Si bien Kant no incurre en los excesos de tantos clásicos del derecho de gentes, algo especialmente notable si se atiende al juicio que le merecerá el colonialismo europeo en América, África y Asia a finales de los años 90, tampoco cabe reconocer en sus escritos una defensa de la protección internacional de los individuos, con independencia de su pertenencia o no a un Estado. Su sistema jurídico no permite localizar instancias jurídicas constitutivas y dotadas de legitimidad de acción a escala global y supraestatal, a pesar de que el conjunto de los Estados se vean beneficiados si se contemplan a la luz de un horizonte federal asintótico, que les devuelve la promesa de la paz perpetua, el único fin final del derecho ${ }^{24}$. Justamente por ello he intentado abogar en este trabajo a favor del carácter regulativo de la mayoría de las tesis y afirmaciones kantianas generalmente asumidas como reivindicaciones de los derechos del hombre, de suerte que su función parece consistir en contribuir a que los Estados asuman el significado y límites del control que ejercen sobre sus propios territorios ${ }^{25}$, por cuanto la posesión común que originariamente todos los seres humanos tienen sobre el planeta debería suponer en todo caso -especialmente en casos límite- una 'razón' suficiente para abrir sus fronteras a la visita de extranjeros, sin declararles de antemano hostilidad alguna. De ese modo, la consistencia cosmopolita del mundo se encargará de recordar constantemente

\footnotetext{
${ }^{24}$ MS, RL, “Conclusión", AA 06: 355: "Puede decirse que este establecimiento universal y duradero de la paz no constituye solo una parte, sino la totalidad del fin final de la doctrina del derecho dentro de los límites de la mera razón; porque el estado de paz es el único en el que están garantizados mediante leyes lo mío y lo tuyo, en un conjunto de seres humanos vecinos entre sí, por tanto, que están reunidos en una constitución". Trad. citada.

${ }^{25}$ En esta línea de actualización del cosmopolitismo kantiano ha avanzado de manera consistente Ypi (2014), cuyas conclusiones deberían contrastarse con las solventes críticas de Pinheiro Walla (2014).
} 
a los poderosos señores de la Tierra que su poder territorial no demarca ninguna frontera definitiva sobre el planeta, pues es más bien la finitud del mismo el único límite extremo que cabe reconocer en él. A mi entender $-\mathrm{y}$ con ello me distancio de propuestas sugerentes como la de Huber- Kant no revisa en ningún momento, ni tampoco da pie para ello en sus textos, la estructura del derecho político, para adaptarlo al punto de vista de los hijos de la Tierra (Erdensohn), como se denominara a sí mismo Kant en una carta a Hamann en abril de 1774, a pesar de que algunos de sus escritos demarquen un espacio jurídico más amplio que el cubierto por los respectivos Estados, coincidente justamente con el espacio físico en que los seres humanos han de poder circular con libertad, sin necesitar de autorización alguna para proponer establecer relaciones comerciales con individuos de otros pueblos.

Esta evidencia obliga a distinguir con cuidado la concepción fundamentalmente moral que Kant tiene de la dignidad humana con respecto al paradigma manejado por los teóricos contemporáneos de los derechos humanos, para los que basta con atender a semejante concepto para comprender que hay toda una lista de derechos que deben ser cuidadosamente protegidos por los Estados, siendo tutelado tal proceso por organismos internacionales. ${ }^{26}$ Frente a ello, no hay signos en Kant de una enmienda al punto de visto estatal como el supremo a efectos de agencia política en el campo del derecho. La presencia de los habitantes de la Tierra que circulan por ella debe producir en el gobernante un sentido de la responsabilidad que le obliga a mirar más allá del territorio que debe administrar y gobernar, poniéndose en el lugar de cualquier otro, que Kant considera siempre como la estructura más básica de toda reflexión y, así pues, de todo uso de la razón. Dicho de otra manera, el derecho cosmopolita señala a los gobernantes que no están solos en el mundo, junto a la ciudadanía que les reconoce como soberanos legítimos, sino que deben comportarse con respeto hacia las necesidades de otros seres humanos, especialmente en situaciones críticas para estos, ofreciéndoles auxilio y protección, a pesar de que nada les obligue a proporcionarles asilo y residencia. Pero esto no comporta que una idea racional como la federación internacional pueda reclamar un derecho a hacerse cargo en todos los sentidos de los seres humanos no protegidos por ningún Estado. Basta recordar que la idea kantiana de federación no afecta, al menos expresamente, a la protección de ningún individuo aislado o en grupo, sino a la relación jurídica que los distintos Estados están llamados a mantener unos con otros, dejando a un lado el vínculo salvaje de la guerra. Tampoco pudo conocer Kant lo que hoy en día calificamos como 'catástrofes humanitarias', esto es, crisis de tal intensidad que invitaran a revisar la centralidad política de la autoridad estatal.

De la misma manera que la exigencia kantiana de respetar a otros seres humanos procede de la ley moral que cada sujeto escucha en su interior, esto es, de la autonomía de su propia razón, el derecho cosmopolita debería considerarse en Kant como una suerte de corolario del derecho público, que aspira a cubrir progresivamente la totalidad del mundo ${ }^{27}$. Así, aunque el gobernante de un Estado no esté obligado a cumplir una ley que imponga proteger efectivamente al conjunto de los seres humanos, la certeza de que la transgresión de la justicia en cualquier rincón de la Tierra se deja sentir en cualquier otro es subrayada por el propio Kant, pero no como una evidencia que traiga consigo la exigencia de establecer nuevas instancias jurídicas, supraestatales, sino más bien con vistas a fortalecer y extender precisamente el régimen republicano estatal sobre el planeta. Pues la realidad

\footnotetext{
${ }^{26}$ Es de justicia señalar que la mayor parte de los trabajos reunidos por Follesdal y Maliks (2014) no encuentran las mismas dificultades que yo a propósito de las virtualidades del pensamiento kantiano para promover una fundación de los derechos humanos, por lo que en ellos encontrará provechosas pautas el lector interesado en esta dirección.

${ }^{27}$ Las dificultades para acuñar una expresión del tipo 'cosmopolitismo moral' en Kant han sido expuestas con rigor por Kleingeld (2016: 15): 'La mayor parte de los lectores (...) asociarán el cosmopolitismo moral kantiano con su tesis de que debemos contemplarnos a nosotros mismos y a los demás como miembros de un 'reino de los fines' moral. Sin embargo, el lenguaje de una 'ciudadanía' (mundial) está manifiestamente ausente de su discusión en la Crítica de la razón pura del 'mundo moral', en el que los agentes morales viven juntos bajo leyes (A808/B836). Kant hace claramente un uso metafórico de un modelo político para evocar el ideal de una comunidad moral mundial de agentes, si bien raramente se refiere a los agentes morales como "ciudadanos' del mundo en semejante mundo moral".
} 
efectiva de lo regulativo, como el mismo Kant indica en el Apéndice a la dialéctica trascendental de la primera Crítica, procede siempre de lo que el entendimiento y el Juicio sean capaces de realizar contando con esos exigentes ideales ( $\mathrm{KrV}$ A657/B685). Asimismo, la idea de una constitución política perfecta no debe desaparecer nunca de la agenda de los soberanos, pero no por ello cabe esperar que ninguno la encarne a la perfección (KrV A316/B372). Merecería así la pena profundizar en la importancia que Kant concede a lo regulativo, a los principios no coactivos y al sentimiento en el interior de lo que entiende como una parte del sistema jurídico, pues ahí encontraremos el nivel de discurso más armonioso con los fines que actualmente se propone la reflexión sobre la justicia global. No debería rebajarse la importancia de tales principios 'blandos', por así decir, o 'vagos' término que Kant emplea también en la primera Crítica (A680/B708)-, puesto que la perspectiva que adoptan es la de nuestra condición finita y corpórea, sino que lo metodológicamente fructífero sería distinguir con rigor su estatuto con respecto al tipo de subjetividad y de justificación indispensable para hacerse cargo de la complejidad de las concepciones contemporáneas de los derechos humanos. ${ }^{28}$

\section{Bibliografía}

AJEI, M. y FLIKSCHUH, K: “Colonial Mentality: Kant's Hospitality Right Then and Now”, en FLIKSCHUH, K. y YPI, L. (eds): Kant and Colonialism. Historical and Critical Perspectives, Oxford, Oxford University Press, 2014, 221-250.

ARENDT, Hannah: "Es gibt nur ein einziges Menschenrecht", Die Wandlung 4 (1949), 754-770.

ARENDT, Hannah: Los orígenes del totalitarismo, Madrid, Alianza, traducción de Pedro Bravo, 2006.

BEITZ, C.: The Idea of Human Rights, New York, Oxford University Press, 2009.

BEITZ, C.: "What Human Rights Mean", Daedalus, 32, 39, (2003), 36-46.

BENHABIB, S.: Another Cosmopolitanism, Oxford, Oxford U.P., 2008.

BERSTEIN, A.: "The Right of States, the Rule of Law, and Coercion: Reflections on Pauline Kleingeld's Kant and Cosmopolitanism", Kantian Review 19/2 (2014), 233-249.

BERSTEIN, A.: "Kant on Rights and Coercion in International Law: Implications for Humanitarian Military Intervention", Jahrbuch für Recht und Ethik 16 (2008), 57-100.

BYRDM B. S. y HRUSCHKA, J.: Kant's Doctrine of Right. A Commentary, Cambridge, Cambridge U.P., 2010.

CAVAllaR, G.: Kant's Embedded Cosmpolitanism. History, Philosophy and Educations for World Citizens, Berlin/Boston, W. de Gruyter, 2015.

CRUFT, R., MATTHEW LIAO, S. y RENZO, M. (eds): Philosophical Foundations of Human Rights, Oxford U.P., 2015.

DORSEY, D.: "Global Justice and the Limits of Human Rights", Philosophical Quarterly 55 (2005), $562-581$.

FLIKSCHUCH, K.: "Human Rights in Kantian Mode: A Sketch", en CRUFT, R., MATTHEW LIAO, S. y RENZO, M. (eds): Philosophical Foundations of Human Rights, Oxford U.P., 2015, 653-670.

FLIKSCHUCH, K.: "Enthusiastic Cosmopolitanism", en COHEN, A. (ed.), Kant on Emotion and Value, London, Palgrave McMillan, 2014, 265-283.

FLIKSCHUCH, K.: "On the Cogency of Human Rights”, Jurisprudence 2/1 (2011), 17-36.

FLIKSCHUCH, K.: "Kant's Sovereignty Dilemma”, The Journal of Political Philosophy, 18/4 (2010), 469-493.

${ }^{28}$ Una exposición y discusión sistemática de la complejidad a las que nos referimos puede consultarse en Cruft/Matthew Liao/Renzo (2015). 
FOLLESDAL, A. y MALIKS, R. (eds.): Kantian Theory and Human Rights, London, Routledge, 2014.

HUBER, J.: "Cosmopolitanism for Earth Dwellers: Kant on the Right to be Somewhere", Kantian Review (2016), en imprenta.

HUBER, J.: "What Makes Human Rights Political? A Kantian Critique", Zeitschrift für Menschenrechte/Journal for Human Rights 2 (2013), 127-140.

KLEINGELD, P.: Kant and Cosmopolitanism, Cambridge, Cambridge University Press, 2011.

KLEINGELD, P.: "Kant's Moral and Political Cosmopolitanism", Philosophy Compass 11/1 (2016), 14-23.

KLEINGELD, P.: “Kantian Patriotism”, Philosophy and Public Affairs 29/4 (2000), 313-341.

MECKSTROTH, C.: "Could Kant Support Human Rights? Kant's Arguments and Their Contemporary Relevance", APSA Annual Meeting Paper (acceso el 13 de mayo de 2016). $\langle$ http://papers.ssrn.com/sol3/papers.cfm?abstract_id=2301504>

PINHEIRO WALLA, A.: "Common Possession of the Earth and Cosmopolitan Right", Kant Studien, 107/1 (2016), 160-178.

PINHEIRO WALLA, A.: “A Commentary on Anna Stilz, 'Nations, States, and Territory' and Lea Ypi, 'A Permissive Theory of Territorial Rights"”, en CARA, Nine (ed.): Territory and Justice Symposia, 2014 (acceso el 15 de junio de 2016). $<$ http://eis.bris.ac.uk/ plcdib/territory.html>

O'NEILL, O.: "The Dark Side of Human Rights", International Affairs 81/2 (2005), 427-439.

POGGE, T.: "Foreword", en FOLLESDAL, A. y MALIKS, R. (eds.): Kantian Theory and Human Rights, London, Routledge, 2014, xv-xx.

POGGE, T.: "Kant's Vision: Europe, and a Global Federation“, en MERLE, J.-C. (ed.): Globale Gerechtigkeit, Stuttgart, Frommann-Holzboog, 2005, 500-518.

RONZONI, M.: “Global Order: A Case of Background Injustice?", Philosophy and Public Affairs 37/3 (2009), 229-256.

SÁNCHEZ MADRID, Nuria: "L'entrecroisement de la théologie et de l'idée de système dans l'«Histoire générale de la nature et théorie du ciel» : la genèse d'une réflexion athéologique sur le cosmos", en VV.AA., Kant avant la «Critique de la raison pure», Paris, Vrin, 2009, 129-136.

SÁNCHEZ MADRID, Nuria: "Kant's Juridical Cosmopolitanism from the Standpoint of some Recent Global Justice Theories”, Studia Philosophica Kantiana 2 (2014), pp. 18-31.

SÁNCHEZ MADRID, Nuria: "Duty and Coercion in Kant's Republican Cosmopolitanism", Kantovski Sbornik. Kant Studies Journal (2015a), pp. 57-69.

SÁNCHEZ MADRID, Nuria: "Algunas aporías del derecho kantiano. Reforma social y republicanismo cosmopolita", Fragmentos de Filosofía, 13 (2015b), 43-57.

SÁNCHEZ MADRID, Nuria: "El carácter jurídico del cosmopolitismo kantiano: las condiciones de la extensión del derecho a escala global", en NAVARRO CORDÓN, J. M., ORDEN, R. y ROVIRA, R.: Kant en nuestro tiempo. Las realidades en que habitamos, Madrid, Biblioteca Nueva, 2016, 207-220.

SANGIOVANNI, A.: "Why there Cannot be a Truly Kantian Theory of Human Rights", en CRUFT, R., MATTHEW LIAO, S. y RENZO, M. (eds): Philosophical Foundations of Human Rights, Oxford U.P., 2015, 671-689.

SENSEN, O.: Kant on Human Dignity, Berlin/Boston, W. de Gruyter, 2011.

TASIOULAS, J.: "Taking Rights out of Human Rights", Ethics 120 (2010), 647-678.

YPI, Lea: “A Permissive Theory of Territorial Rights", European Journal of Philosophy 22 (2014), 288-312.

WILLIAMS, H.: "Kantian Underpinnings for a Theory of Multirights", en FOLLESDAL, A. y

MALIKS, R. (eds.): Kantian Theory and Human Rights, London, Routledge, 2014, 8-26. 